\title{
Gold Black and Gold Cermet Absorbing Surfaces
}

\author{
David R. McKenzie
}

School of Physics, University of Sydney, Australia

Gold black and gold cermet coatings present interesting properties for radiation detection and solar energy conversion applications. The characteristics required for each specific application can be obtained by taking advantage of the critical dependence of the properties of such coatings upon the conditions used for their preparation.

It has been known for some time that metals may be evaporated from a hot wire in gas at a pressure of the order of $200 \mathrm{~Pa}$ to produce black coatings which consist of fine particles. The coatings produced using gold, gold blacks, are the best known. The apparatus used by Pfund $(1,2)$, one of the first to produce metal blacks, is illustrated in Figure 1. The gold is placed in the tungsten filament $F$ which is electrically heated. The gold vapour thus generated is chilled by the gas in the chamber and condenses to form tiny crystals which are carried by convection currents to coat nearby objects. Coatings do not form equally heavily on all substrates; the crystals tend to avoid thick dielectric substrates presumably because of charge build-up.

Harris (3) found that gold blacks are extremely tenuous coatings with densities that may be as low as 0.002 times that of bulk gold. A good impression of the morphology and tenuous nature of gold black coatings is given by Figure 2 where characteristic crystals of gold, of roughly bipyramidal shape, can be seen linked together to form a network. The size of the crystals depends on such parameters as the rate of evaporation, the pressure and properties of the scattering gas, and the source to substrate distance (4). For instance, with helium as the scattering gas, the crystallite size varies from 5 to $10 \mathrm{~nm}$ and the packing fraction of gold varies from 0.03 to 0.003 as the pressure is increased from 60 to $1330 \mathrm{~Pa} \mathrm{(5).}$

The visible light reflected from a gold black coating is essentially all diffuse (3) and is of the order of 1 per cent of the incident light for a coating of about $1.5 \mathrm{~g} / \mathrm{cm}^{2}$. When coated on to bright gold the total reflectance of the combination varies from about 1.6 per cent at $0.40 \mu \mathrm{m}$ wavelength to about 3.1 per cent at $2.2 \mu \mathrm{m}$ for a coating of about $1 / \mathrm{gm}^{2}$ prepared in pure nitrogen (6). For the same coating, the reflectance becomes more specular in the infrared but the absorptance is greater than 97 per cent in the region up to $15 \mu \mathrm{m}$ wavelength.

Because of its high absorption properties and low thermal mass, gold black is used as a coating

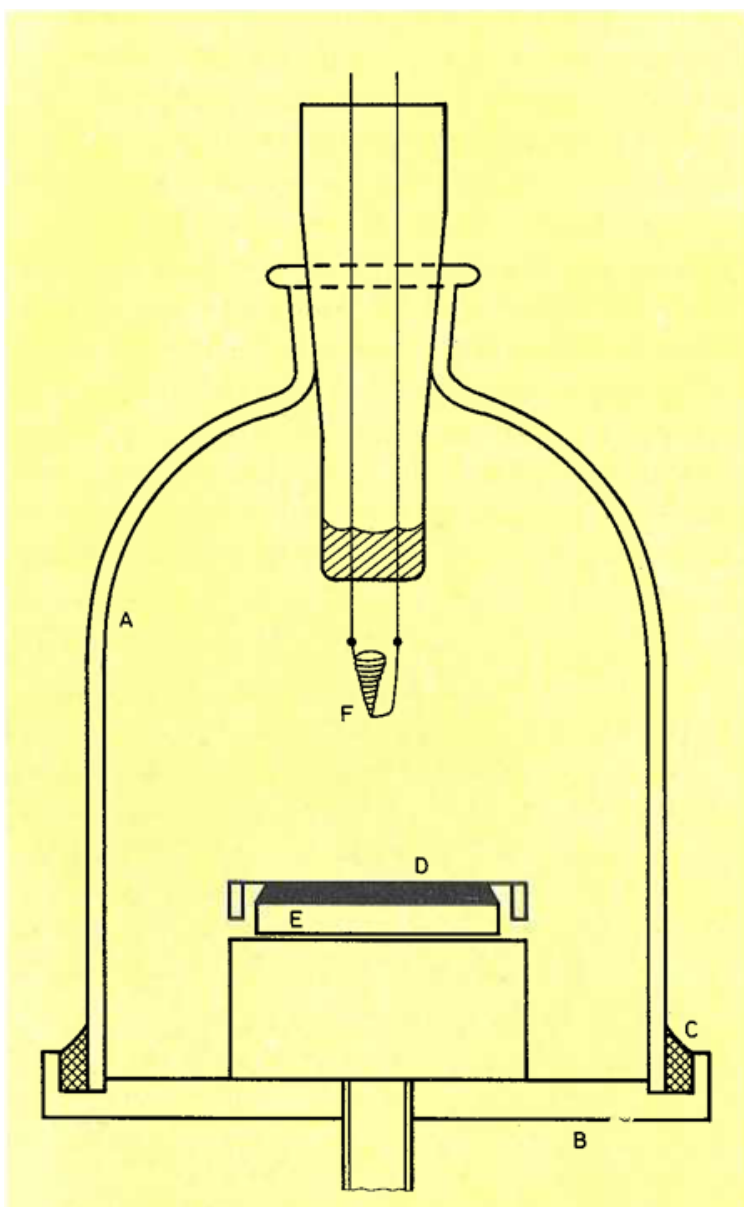

Fig. 1 The apparatus of Pfund $(1,2)$ for the production of metal blacks. $F$ is a tungsten spiral into which the metal to be evaporated is placed. The substrate film D is stretehed over a mercury surface $\mathbf{E}$ 


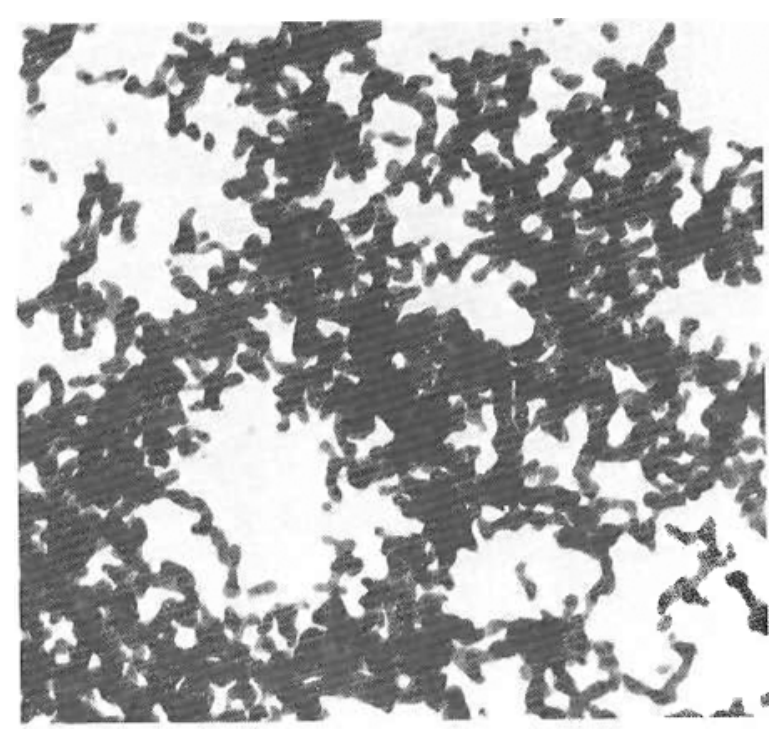

on radiation detectors of high sensitivity and fast response. Harris (3) has found that a coating of $0.75 \mathrm{~g} / \mathrm{cm}^{2}$ gives optimum performance in the range 1 to $15 \mu \mathrm{m}$ for detectors in thermopiles. Heavier coatings, although increasing the absorptance, damage the response time because of their thermal mass and relatively poor thermal conductance. Bolometer receivers also have an optimum coating thickness at about this value. Pyroelectric detectors are not nearly as sensitive to the thermal mass of the black coating; a coating of 2 to $6 \mathrm{~g} / \mathrm{m}^{2}$ gives optimum performance for a detector using Tedlar polyvinyl fluoride film (7). Black paint, however, has a thermal mass two orders of magnitude higher than gold black and actually lowers the performance of the coated detector at low frequency, relative to the uncoated detector.
Fig. 2 Gold black viewed in the transmission electron microscope. This coating was prepared in $133 \mathrm{~Pa}$ of nitrogen. The crystals are approximately $10 \mathrm{~mm}$ in diameter

\section{Gold Smoke Deposits}

If the gas in which the evaporation is made is not completely free of oxygen, a coating is produced which is superficially similar to normal gold black but does not have the wide-band absorption property. Coatings prepared, for example, in nitrogen containing 20 per cent oxygen at $400 \mathrm{~Pa}$ become almost transparent for wavelengths longer than $2 \mu \mathrm{m}(8,9)$. These coatings are termed gold smoke deposits to distinguish them from the normal gold black.

Figure 3 provides a comparison of the reflectance of a copper surface coated with gold blacks prepared in various gases. Curves $\mathrm{c}$ and $\mathrm{d}$ are for gold smokes and display the type of behaviour required from selective absorbers for the collection of solar energy. The requirement for this application is a surface of high absorptance for wavelengths less than about $2 \mu \mathrm{m}$ and low emittance for wavelengths longer than this value. Surfaces of solar absorptance 0.88 for normal incidence with emittance 0.10 for room temperature radiation can be obtained using gold smokes (9). The dip in reflectance at a wavelength of around $12 \mu \mathrm{m}$ (see curves $\mathrm{c}$ and d in Figure 3) is somewhat damaging to the low emittance property and has been shown to be due to the presence in the coatings, of oxides of tungsten $(3,9)$ produced by oxidation of the evaporation filament. The tungsten oxide enters intimately into the chain structure of the gold crystallites as established (9) by imaging a

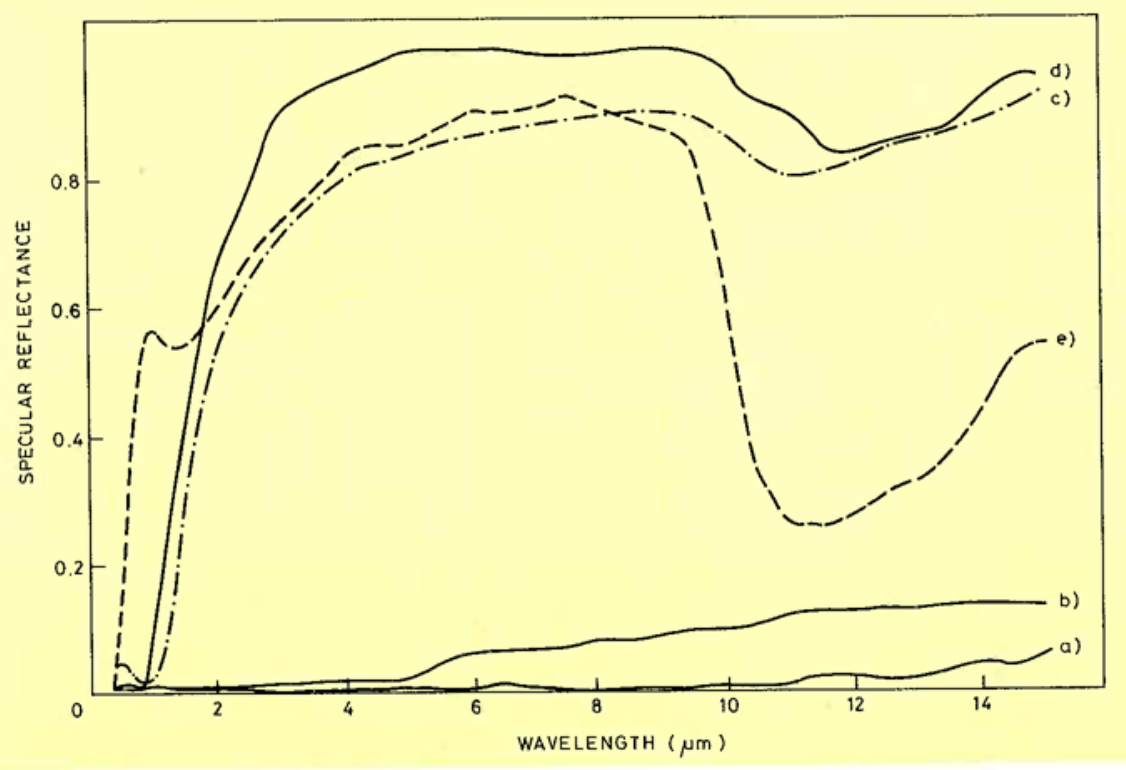

Fig. 3 Specular reflectance of various gold black and gold smoke coatings on a copper substrate (9):

a) gold black prepared in oxygen free nitrogen at 400 $\mathrm{Pa}$, mass thickness $0.81 \mathrm{~g} / \mathrm{m}^{2}$ b) gold black prepared in nitrogen containing 5 per cent oxygen at $400 \mathrm{~Pa}$, mass thick. ness $0.90 \mathrm{~g} / \mathrm{m}^{2}$

c) gold smoke prepared in nitrogen containing 20 per cent oxygen at $400 \mathrm{~Pa}$, mass thickness $0.98 \mathrm{~g} / \mathrm{m}^{2}$

d) gold smoke prepared in nitrogen containing 20 per cent oxygen at $1300 \mathrm{~Pa}$, mass thickness $0.98 \mathrm{~g} / \mathrm{m}^{2}$ e) tungsten oxide deposit prepared in pure oxygen at $400 \mathrm{~Pa}$, mass thickness 0.97 $\mathrm{g} / \mathrm{m}^{2}$ 
sample of gold smoke in the electron microscope using one of the diffraction rings of tungsten oxide (dark field microscopy). The insulating crystals of tungsten oxide interrupt the conducting gold chains and cause a very large increase in the electrical resistivity of the coatings, $(3,9)$ as well as the change in optical properties.

There are, however, two disadvantages of gold smokes as absorbing surfaces for solar collectors. The first is the very low resistance to abrasion: both gold black and gold smoke deposits are easily damaged by mechanical contact as well as humidity. The second is the tendency to sinter when held at temperatures above $69^{\circ} \mathrm{C}$ (3). In the process, the tungsten oxide crystals are excluded from their positions between gold crystals thereby causing the chains of crystals to become conductive. The optical and electrical properties of a gold smoke sintered at $170^{\circ} \mathrm{C}$ for 3 hours and $180^{\circ} \mathrm{C}$ for 54 hours are almost indistinguishable from those of a normal gold black of similar areal density (3). Normal gold black deposits themselves sinter when held at temperatures above $100^{\circ} \mathrm{C}$, first to a brownish coloured coating, then to a yellow gold film (3). The gold black sintering process is somewhat less rapid if the coating is stabilized at $69^{\circ} \mathrm{C}$ for 24 hours. This stabilization is recommended for gold blacks intended for use in radiometers.

\section{Optical Theory of Gold Black and Gold Smoke}

The extreme blackness of gold black over a wide spectral region is essentially caused by the wide distribution of effective particle sizes, ranging from
$5 \mathrm{~nm}$ to about $8 \mu \mathrm{m}$ (10). Light of wavelengths $\lambda$ ranging from the visible to about $50 \mu \mathrm{m}$ finds conducting particles of dimension approximately $\lambda / 2 \pi$ with which it interacts strongly and is largely absorbed or scattered. The smallest particles in the coating are the gold crystallites themselves and the larger particles are chains of crystallites. Zaeschmar and Nedoluha (11) have developed an impedance network analogy for gold black which, although neglecting scattering, simulates the behaviour of gold black over a wide wavelength band. For wavelengths longer than $50 \mu \mathrm{m}$ the coating appears as a smooth conducting film and has appreciable reflectance

Variation of the oxygen content of the scattering gas produces a range of coatings whose absorption region varies in width. The coatings made in the highest oxygen content have the narrowest absorption region. The gold crystals are then isolated from each other by insulating material and have a size much less than the wavelength of light. The simple treatment of Maxwell-Garnett $(12,13)$ is then applicable and gives reasonable agreement for these coatings $(9,14)$. The Maxwell-Garnett relation for the relative permittivity $\varepsilon$ of a dilute suspension in air of spheres of material of relative permittivity $\varepsilon^{\prime}$ with volume fraction $\mathrm{f}$ is :

$$
\frac{(\varepsilon-1)}{(\varepsilon+2)}=\mathrm{f} \frac{\left(\varepsilon^{\prime}-1\right)}{\left(\varepsilon^{\prime}+2\right)}
$$

The absorption minimum predicted by the theory is, however, generally sharper than the measured curves. The reason for this may be due to one or more of the following $(15,16)$ :
Fig. 4 Three specimens of selective gold-alumina cermet films produced by evaporation in high vacuum from an electron beam-heated source of alumina and a resistively-heated source of gold

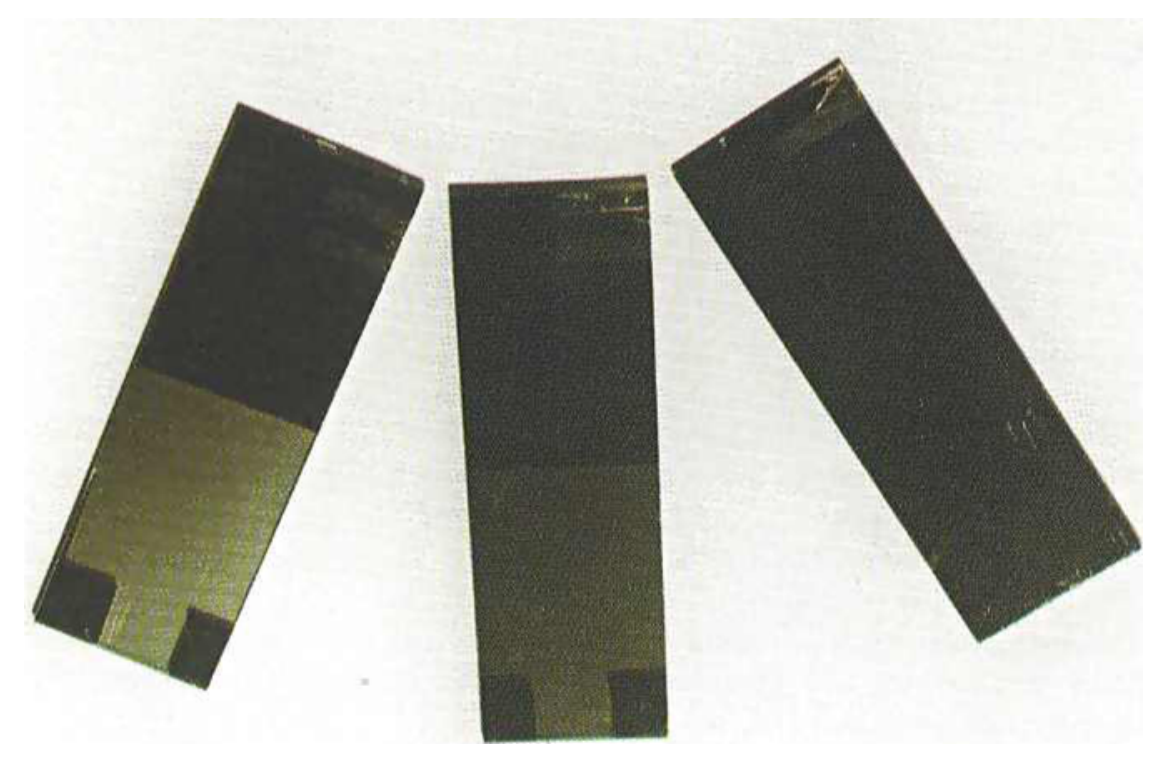




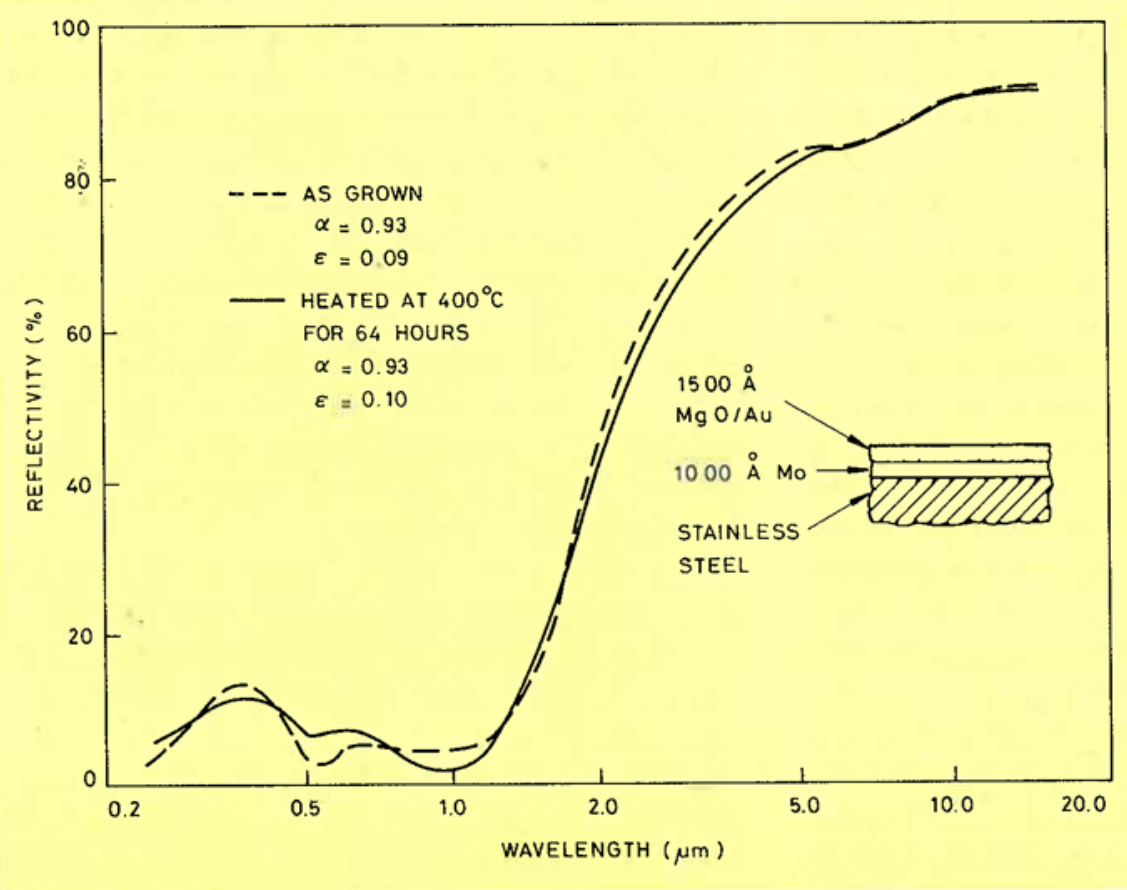

Fig 5 Integrated spectral reflectance of as-grown and heat-treated gold-magnesia cermet films deposited onto molybdenum-coated stainless steel. The coating method was radio-frequency sputtering from a composite target. The solar absorptance and infrared emittance are respectively $\alpha$ and $\varepsilon$. After Fan and Zavracky (18)

(1) A variation of the effective optical constants of gold with particle size

(2) The effects of interaction between particles

(3) The effect of an oxide coating on the particles

Coatings made in low oxygen-containing gas mixtures have broader absorption regions owing to the contribution of scattering (9). Some insight is gained by the application of the theory of Mie (17) for scattering and absorption by spheres. The effective particle sizes may be determined by fitting the experimental curves.

\section{Gold Cermets}

A dispersion of gold particles is made more stable against sintering by using a ceramic material as the dispersing medium rather than air or vacuum. Such dispersions may be produced by evaporation in high vacuum of a metal from one source and the ceramic from another (Figure 4). An alternative method of production is radiofrequency sputtering from a composite target. These ceramic-metal alloys or cermets have properties intermediate between metals and nonmetals. Gold cermet coatings are generally adherent to metal substrates and resistant to abrasion. When applied to a metal substrate in the correct thickness and composition range, they provide a surface of high solar absorptance and low thermal emittance $(18,19)$.

Measurements have been made of the optical constants of cermets of gold with alumina (20), magnesia (18), magnesium fluoride (21) and silica (22) with gold volume fractions less than 50 per cent. The reason for the high absorptance property of cermet coatings on metal is simply that the optical constants lie in the region where the interference annulment for a quarter-wavelength film is high over the solar spectrum $(23,24)$. Electron microscopy (19) of a typical cermet film of 25 per cent gold volume fraction shows that the gold particles are of the order of $10 \mathrm{~nm}$ in diameter. Scattering should therefore play no part in the optical properties but the elementary MaxwellGarnett theory or its extension to non-spherical particles by Galeener (25), do not explain the significant value of the absorption index in the near infrared $(19,20)$ which is essential to the high solar absorptance property.

This failure of the Maxwell-Garnett formula is understandable because of the failure of the assumption that the spheres are sufficiently separated to be considered immersed in a uniform field. The effect of interaction between particles is to cause the induction of multipole moments on the gold particles $(26,27,28)$. This leads to enhanced infrared absorptance $(20,27)$. Additional effects such as the variation of the optical constants of gold with particle size, the deviation from spherical shape, the possiblity of electron tunnelling between particles, and the retardation of the electromagnetic field (29) may also need to be taken into account. 
The absorptance of a single layer film may be increased by grading the composition of the film from the substrate to the surface in such a way that the optical constants vary from values characteristic of metals to values characteristic of transparent insulators (23). This may be done readily in a gold cermet film by decreasing the volume fraction of gold continuously towards the surface. Gold cermet absorbers of absorptance 0.94 and emittance 0.03 have been made in this way (28). Furthermore, gold cermets appear to be stable in air at temperatures up to $400^{\circ} \mathrm{C}$ (18) and in vacuum at temperatures up to $300^{\circ} \mathrm{C}(18,28)$. This stability is in part due to the general resistance of gold to chemical reaction so that the two phases nature of the cermet is preserved. Figure 5 shows the reflectance wavelength profile of a gold cermet before and after ageing.

The properties of highly selective surfaces such as gold cermet absorbers are best used in collectors which employ insulation by vacuum The collector array in Figure 6, developed at the University of Sydney (30), consists of tubular glass absorber tubes coated with a selective surface of absorptance 0.85 and emittance 0.03 (at room temperature) and surrounded by an evacuated glass envelope. Collectors such as this are capable of delivering heat at temperatures up to $300^{\circ} \mathrm{C}$ without the need for concentration of sunlight or the need for steering. They are potential suppliers of low and medium grade heat for industrial processes as well as for domestic hot water.

\section{Acknowledgements}

The author thanks Drs. Granqvist, Doland, O'Neill and Ignatiev for their assistance in preparing this review, Dr. B Window for permission to use the photograph of the University of Sydney solar collector and Professor H. Messel and the Science Foundation for Physics for their support.

\section{References}

1 A. H. Pfund, Rev. Sci. Inst., 1930, 1, 397

2 A. H. Pfund, F. Opt. Soc. Am., 1933, 23, 375

3 L. Harris, "The Optical Properties of Metal Blacks and Carbon Blacks', The Eppley Foundation for Research, Newport, Monograph Series No. 1, 1967

4 L. Harris, D. Jeffries and B. M. Siegel, F. Appl. Phys., 1948, 19,791

5 C. Doland, P. O'Neill and A. Ignatiev, F. Vac. Sci. Technol., 1977, 14, 259

6 R. G. Johnston and R. P. Madden, F. Appl. Opt., 1965, 4, 1574

7 W. R. Blevin and J. Geist, F. Appl. Opt., 1974, 13, 1171 8 L. Harris and J. K. Beasley, f. Opt. Soc. Am., 1952, 42, 134

9 D. R. McKenzie, 7. Opt. Soc. Am., 1976, 66, 249

10 L. Harris and K. F. Cuff, F. Opt. Soc. Am., 1956, 46, 160

11 G. Zaeschmar and A. Nedoluha, 7. Opt. Soc. Am., 1972 , 62,348

12 J. C. Maxwell-Garnett, Phil. Trans. R. Soc., (London), $1904,203,385$

13 J. C. Maxwell-Garnett, Phil. Trans. R. Soc., (London), 1906, 205,237
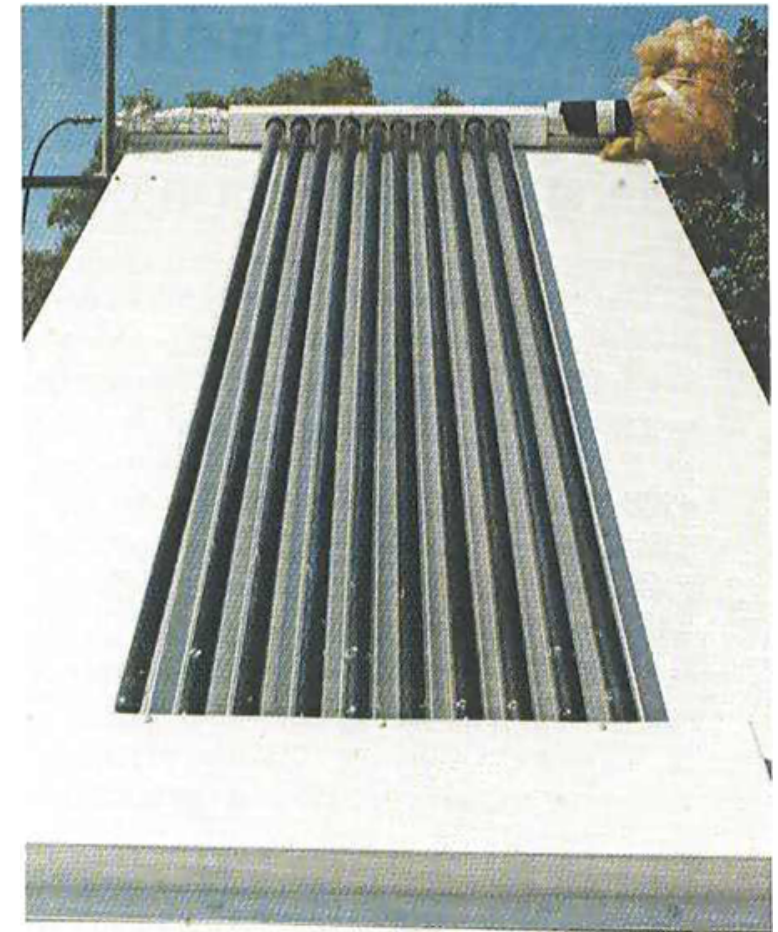

Fig. 6 The prototype solar collector developed at the University of Sydney. The ten collector tubes are vacuum insulated and have a selective coating applied to them. The collector is shown producing steam at $100{ }^{\circ} \mathrm{C}$

14 C. G. Granqvist and O. Hunderi, Phys. Rev. B., 1977, (in press)

15 C. G. Granqvist and O. Hunderi, Solid State Conmun., $1976,19,939$

16 C. G. Granqvist, 'Conference on Electrical, Transport and Optical Properties of Inhomogeneous Media', Ohio State Univ., Columbus, Ohio, 7-9 September 1977, (to appear in A.I.P. Conf. Ser.)

17 G. Mie, Ann. Phys., 1908, 25, 377

18 J. C. C. Fan and P. M. Zavracky, Appl. Phys. Lett., 1976 29, 478

19 J. I. Gittleman, B. Abeles, P. Zanzucchi and Y. Aris, Thin Solid Films, 1977, 45, 9

20 D. R. McKenzie and R. C. McPhedran, 'Conference on Electrical, Transport and Optical Properties of Inhomogeneous Media', Ohio State Univ., Columbus, Ohio, 7-9 September 1977, (to appear in A.I.P. Conf. Ser.),

21 P. H. Lissberger and R. G. Nelson, Thin Solid Films., 1977 21, 159

22 R. W. Cohen, G. D. Cody, M. D. Coutts and B. Abeles, Phys. Rev. B., 1973, 8, 3689

23 I. T. Ritchie and B. Window, Appl. Opt., 1977, 16, 1438

24 D. R. McKenzie, Appl. Opt., 1978, (in press)

25 F. L. Galeener, Phys. Rev. Lett., 1971, 27, 421

26 J. W. Rayleigh, Phil. Mag., 1892, 34, 481

27 W. T. Doyle, 'Conference on Electrical, Transport and Optical Properties of Inhomogeneous Media', Ohio State Univ., Columbus, Ohio, 7-9 September 1977, (to appear in A.I.P. Conf. Ser.)

28 D. R. McKenzie, unpublished work

29 C. G. Granqvist and O. Hunderi, Phys. Rev. B, 1977, (in press)

30 Annual Report, Energy Research Centre, University of Sydney, 1976 